\title{
CESR Phase III Interaction Region *
}

\author{
S. Henderson, J. J. Welch ${ }^{\dagger}$, M. Billing, G. Cherwinka, G. Codner, G. Dugan, S. Greenwald, \\ Z. Greenwald, Y. Li, W. Lou ${ }^{\ddagger}$, N. Mistry, E. Nordberg, D. Rice, S. Roberts, D. Rubin, \\ A. Temnykh, Cornell University, Ithaca NY \\ D. Cinabro, L. Perera, Wayne State University, Detroit MI
}

The goal of the third phase of the CESR/CLEO upgrade is to take the CESR luminosity as far as one can go with a single-ring collider [1]. We expect CESR's luminosity to more than double from the present $8 \times 10^{32} \mathrm{~cm}^{-2} \mathrm{~s}^{-1}$ to $1.7 \times 10^{33} \mathrm{~cm}^{-2} \mathrm{~s}^{-1}$ as a result of the Phase III upgrade.

In the present configuration of CESR (Phase II) trains of closely spaced bunches collide at a small horizontal crossing angle $\left(\theta_{c} \simeq \pm 2 \mathrm{mrad}\right)$. The Phase III upgrade program relies on increasing the colliding beam current from the present $0.5 \mathrm{~A}$ to $1 \mathrm{~A}$ by exploiting bunch-train collisions to the fullest extent possible. The Phase III design calls for colliding 9 trains of bunches, each of which consists of 5 bunches spaced by $14 \mathrm{~ns}$.

The closely spaced bunches in a train generate crossing points close to the interaction point (IP) where bunches from opposing beams pass by one another and interact. To ameliorate the destructive effects of these long range beam-beam interactions new interaction region (IR) optics are required. A combination of short focal length and large physical aperture are optimum in this respect, and best implemented with insertion optics consisting of two pairs of high-gradient superconducting (SC) quadrupoles and a pair of short permanent magnets. The IR quadrupoles will be positioned with a precision beam-based alignment system to ensure stable closed orbits and reduce commissioning time. The SC magnet package also includes skew quadrupole magnets for detector solenoid compensation and dipole magnets to provide precise alignment of the magnetic centers within the physical aperture of the vacuum chamber.

In order to handle the increased heat loads and detector backgrounds new detector shielding and IR vacuum chambers are being constructed. The design of the machinedetector interface was based on our experience with the CESR Phase II IR [2]. In order to facilitate installation and maintainence a remotely actuated vacuum seal called the "magic flange" has been developed and built. We plan to install the Phase III IR hardware in mid-2000.

The new interaction region is ideal for various possible future upgrade paths which could take CESR into the $10^{34} \mathrm{~cm}^{-2} \mathrm{~s}^{-1}$ luminosity range. For example, a two-ring upgrade [3] would use the large aperture for a crossing angle. An upgrade involving smaller $\beta^{*}$ would benefit from the short focal length since the peak beta function and chro-

\footnotetext{
* Supported by the National Science Foundation

† welch@lns.cornell.edu

${ }^{\ddagger}$ presently at ADC Telecommunications, CT
}

maticity are thereby reduced. Finally, space for an additional magnet for a round beam insertion optics has been provided as well.

\section{OPTICS}

The Phase III IR optics, shown in Figure 1, accomodate the various constraints that arise from the required beam separation in the IR, injection considerations and beamgenerated detector background requirements.

As mentioned, the challenge for operating with $14 \mathrm{~ns}$ spacing between bunches in a train is providing adequate separation at the nearby parasitic crossing points in the IR (spaced at $2.1 \mathrm{~m}$ intervals). In order to minimize the longrange beam-beam tuneshifts suffered at these crossings the beam separation to horizontal width ratio must be maximized, and the $\beta$-functions at the crossing points must be minimized. The latter feature is accomplished by placing the final-focus quadrupoles as close to the IP as possible so that the $\hat{\beta}$ points are reduced and lie between the IP and the first parasitic crossing point. This solution, made possible only with high-gradient SC quadrupoles, maintains the $\beta$-functions at the IR crossing points below $40 \mathrm{~m}$, a value which is comparable to that at crossing points in the CESR

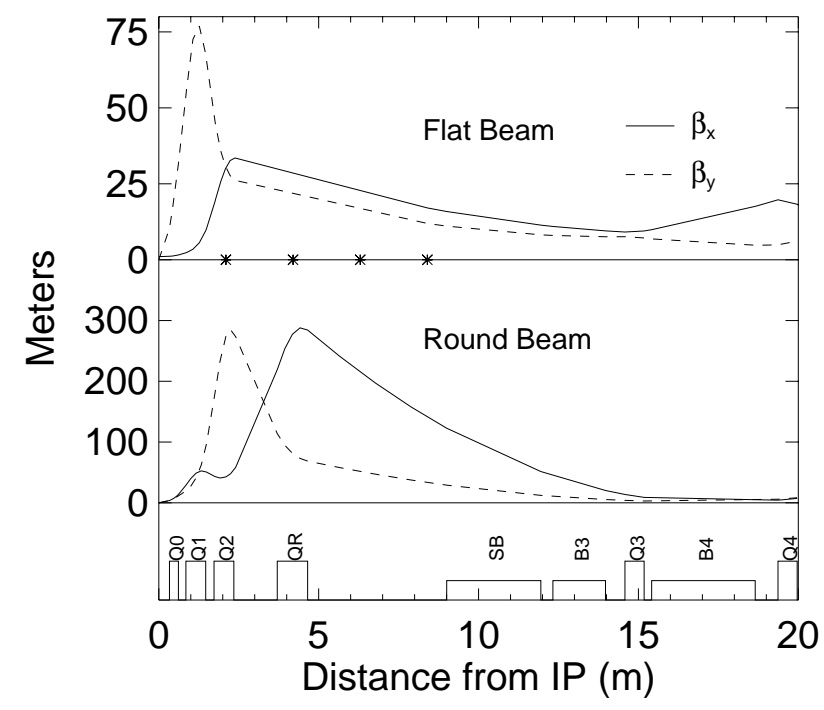

Figure 1: CESR Phase III IR optics with $\beta_{v}^{\star}=10 \mathrm{~mm}$ (upper) and round-beam optics (lower). Parasitic crossing point locations are marked with a star. The trim quadrupole (QR) is powered only in the round beam optics. 


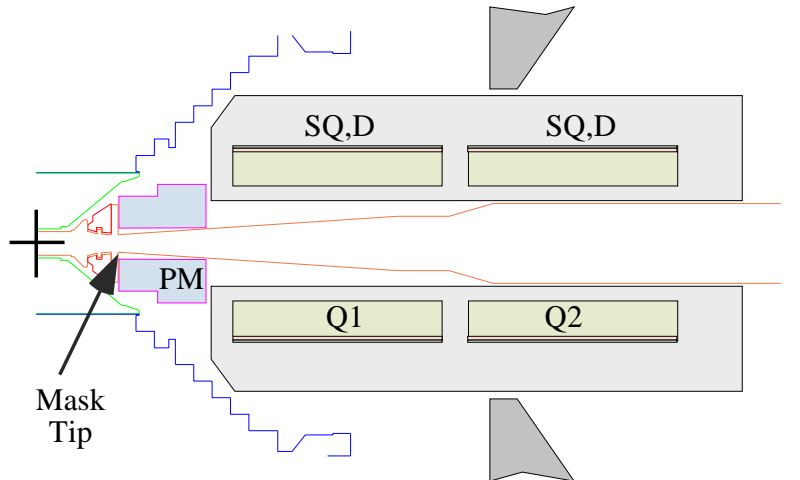

Figure 2: IR layout showing 7 magnetic elements.

arcs.

Constraints on the machine aperture in the IR arise from injection and detector background considerations. During injection the beams are separated horizontally at the IP with a symmetric electrostatic displacement bump. As a result, increased aperture at Q2 is required. In a crossing-angle orbit, the beam is displaced in the nearby horizontally focusing IR quadrupole (Q2). This off-axis beam generates large synchrotron radiation (SR) fluxes which give rise to detector backgrounds. To minimize backgrounds, a horizontal IP displacement bump is applied, reducing the displacement in Q2 at the expense of increased aperture requirements.

The high-gradient quadrupoles provide considerable flexibility in lattice design. For example, considerable latitude is allowed in choosing $\beta_{v}^{\star}$ for optimizing luminosity. Figure 1 shows IR optics for $\beta_{v}^{\star}=10 \mathrm{~mm}$ but optics with $\beta_{v}^{\star}$ as small as $7 \mathrm{~mm}$ are possible. Further increases in luminosity may be possible with round colliding beams [4]. With the addition of a trim quadrupole, round-beam optics with $\beta_{v}^{\star}=\beta_{h}^{\star}=3 \mathrm{~cm}$, shown in Figure 1, may be realized.

\section{INTERACTION REGION MAGNETS}

In total there are 14 different magnets arranged symmetrically around the IP that form the insertion optics (see Figure 2). Starting only $337 \mathrm{~mm}$ from the IP, vertical focussing (VF) is provided by a short set of permanent magnets (PM). Most focussing, however, is provided by two pairs of strong superconducting main quadrupoles (MQ) which are arranged in doublets called Q1,Q2. Skew quadrupoles (SQ) concentrically wound on top of each of the MQ effectively rotate the main quadrupole field as part of the coupling compensation for the detector solenoid. Dipoles (D) concentrically wound on top of the SQ's generate a horizontal field for vertical adjustment of the quadrupole field axes. The entire insertion optics focussing is contained within $\pm 2.55 \mathrm{~m}$ from the IP.

The PM's are made from Neodymium Iron Boron with intrinsic coercivity $H_{c i}$ of $21 \mathrm{kOe}$ and remnant field $B_{r}$ of $12 \mathrm{kG}$ [5]. The ID is a constant $\phi 67 \mathrm{~mm}$ but the OD is stepped to take advantage of slightly more radial space

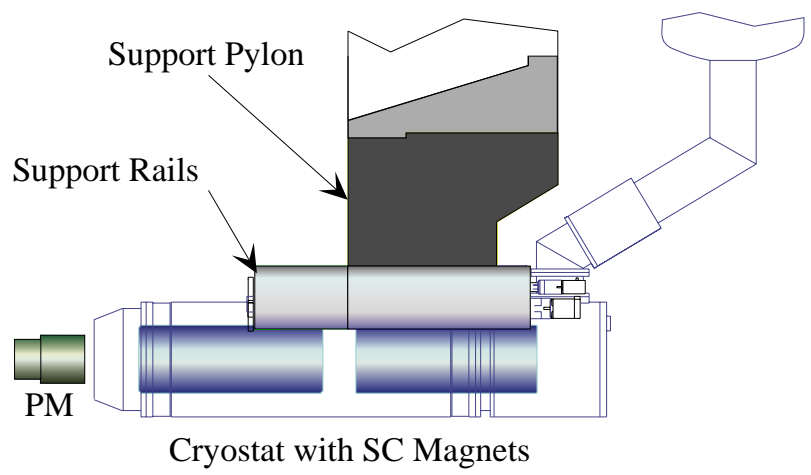

Figure 3: A representation of a cryostat containing two superconducting magnets.

available further from the IP. The poles are temperature and magnetically stabilized and then individually selected for uniformity. Despite a temperature coefficient for $B_{r}$ of $-0.1 \%{ }^{\circ} C^{-1}$, adequate temperature control can be provided with a simple closed circuit cooling loop and bands around the outside of the magnet since the PM only provides about $23 \%$ of the total vertical focussing power.

One cryostat is installed on each side of the IP and contains two multifunctional SC magnets [6]. The cryostats are supported and aligned by eccentrically driven cam bearings held by support rails, which are in turn mounted on a heavy steel pylon attached to the CLEO detector steel. (See Figure 3). The cryostat has a warm bore with an ID of $\phi 145 \mathrm{~mm}$ and a maximum OD of $\phi 495 \mathrm{~mm}$.

All four SC magnets are identical and consist of three independent sets of coils with effective lengths of $0.65 \mathrm{~m}$. The MQ's are capable of gradients of up to $48.4 \mathrm{~T} / \mathrm{m}$ at $1225 \mathrm{~A}$. The SQ field is about $10 \%$ that of the MQ and superimposed on the MQ field. The dipole coils produces a horizontal dipole field with a maximum strength of $0.13 \mathrm{~T}$.

The design of the MQ coils was highly influenced by the recently built LEP 200 Interaction Region quadrupoles $[7,8]$, especially in choice of bore and conductor. The other SC coils are loosely modeled after corrector magnets under development for the LHC. To improve training and reliability the design current of all magnets is no more than $70 \%$ of the theoretical quench current under worst case conditions (multiple coils energized and the presence of the 1.5 T CLEO solenoid field).

Because of the relatively short length compared to diameter, end effects are proportionally more important. Several independent checks were made to be sure the 3D fields from the ends were calculated correctly and the coil design optimized for field quality [9]. The basic specification is that all non-quadrupole and non-dipole field harmonics shall be less than $5 \times 10^{-4}$ of the quadrupole field measured at a radius of $50 \mathrm{~mm}$.

The SC magnets will interact strongly with the detector solenoid magnetic field. Q1 will be totally within the 1.5 $\mathrm{T}$ soleonoid field. The solenoid field and the current in the dipole will generate a very large moment of 12,000 Nm 
on Q1. Q2 will have one end in the solenoid fringe field while the other end is essentially field free, resulting in a net horizontal force on Q2 of up to 14,000 N. These forces and moments must be borne by the cryostats and supports, consequently these constructions are made very robust.

\section{DETECTOR BACKGROUNDS}

Beam-generated detector backgrounds [2] arise from scattered synchrotron radiation (SR) emitted in nearby quadrupoles and bends and from the interaction of beam particles with residual gas in the vacuum chamber ("beamgas" interactions). Shielding of the detector from scattered SR is accomplished by careful placement of mask tips to shield the detector beampipe from direct SR and to minimize the scattered photons which strike the detector. The principal component is a stepped copper mask whose profile is designed such that SR illuminates only those surfaces which are not directly visible by the detector beampipe. Thus, the only remaining detector backgrounds arise from SR which forward scatters through the mask tips ("tipscattering") or which backscatters from the mask surface just downstream of the beampipe. To further reduce the SR flux entering the detector, the inner surface of the beryllium beampipe is coated with a thin layer of gold. The placement of mask tips and choice of materials was optimized with a detailed simulation of SR backgrounds.

Beam-gas backgrounds arise from Coulomb scattering and Bremstrahlung interactions with residual gas. Only those interactions within $\sim 40 \mathrm{~m}$ from the IP contribute to detector backgrounds. Detector shielding for beamgas backgrounds is provided by the PM quads themselves. Additionally, beam-gas backgrounds are reduced with increased pumping in the IR straight.

The detector background simulations provide an estimate of the detector occupancy in the inner layer of the CLEO-III silicon vertex detector. The detector occupancy predictions from the various sources at full $1 \mathrm{~A}$ beam current and $\theta_{c}=2.5 \mathrm{mrad}$ are as follows (in units of $\% / \mu \mathrm{s}$ ): SR - Tipscattering, 0.027 ; SR - Backscattering, 0.012 ; beam-gas Bremsstrahlung, 0.23 ; beam-gas Coulomb, 0.04 . The total estimated occupancy is $0.31 \% / \mu \mathrm{s}$, which is well below the design goal of $1 \% / \mu \mathrm{s}$.

\section{VACUUM SYSTEM}

The central detector beampipe (manufactured by Brush Wellman - Electrofusion Products) is a double-walled actively cooled beryllium beampipe which is based on one presently in use at CESR [10]. The beampipe consists of two Be sleeves of thickness 0.013 in and 0.009 in with a 0.017 in coolant gap. The sleeves are sealed with a manifold at each end. The inner sleeve is joined to a copper vacuum pipe through a stainless steel transition. The beampipe, cooled with PF-200, is capable of dissipating $400 \mathrm{~W}$ with $5^{\circ} \mathrm{C}$ temperature rise.

An important component of the SR shielding is a gold coating on the inside of the beryllium beampipe. A $5 \mu \mathrm{m}$ average thickness coating was applied with a cylindrical magnetron sputtering technique [11]. The total average beampipe thickness is $0.4 \%$ radiation length.

The IR vacuum system utilizes several existing components and new components within $\pm 6 \mathrm{~m}$ from the IP. IR Pumping [12] is accomplished in large plenums which incorporate massive titanium sublimation pumps. Pumping plenums are located at $\pm 3 \mathrm{~m}, \pm 5 \mathrm{~m}, \pm 7 \mathrm{~m}, \pm 10 \mathrm{~m}$ and $\pm 13 \mathrm{~m}$ from the IP. The pressure in the IR will be maintained below $3.5 \mathrm{nTorr}$ at full $1 \mathrm{~A}$ beam current.

The inclusion of a pair of remotely operable ultra-high vacuum joints about $\pm 30 \mathrm{~cm}$ from the IP greatly facilitates the installation and assembly of the IR components. Without this joint, two very difficult field-welds would need to be performed to join UHV flanges to the beryllium beampipe subassembly. The magic flange uses a shaft seal with differentially pumped o-rings (including one which provides the RF seal) in order to eliminate the bulk of the gas load which would arise from permeation. The o-rings have been extensively tested for radiation damage and RF sealing capability. A compact gear-and-nut assembly provides the closing and opening forces in the very small space allowed.

\section{REFERENCES}

[1] D.L. Rubin, Proc. EPAC 1998, http://www.cern.ch/EPAC

[2] S. Henderson, Proc. Second Workshop on Backgrounds at the Machine Detector Interface, World Scientific, p.6 (1998); also S. Henderson, Proc. Part. Acc. Conf. 1997, Vancouver

[3] A. Mikhailichenko and D. Rubin, CLNS-96-1420, http://www.lns.cornell.edu/public/CLNS

[4] E. Young et. al., Proc. Part. Acc. Conf. 1997, 1542.

[5] W. Lou, et. al., Proc. Part. Acc. Conf. 1997,3236, http://www.Ins.cornell.edu/public/CBN/1997

[6] J.J.Welch, et. al., Proc. Part. Acc. Conf. 1997, 3383, http://www.lns.cornell.edu/public/CBN/1997

[7] M. Begg, A. Ijspeert, T.M. Taylor, CERN AT/94-27 (MA), LEP2note 94-14, presented at EPAC 94, (1994)

[8] T.M. Taylor, G. Trinquat, L.R. Williams, IEEE Trans on Magn., vol 28 No 1, pp 382-385, January 1992

[9] G. Dugan, CBN 96-5, http://www.lns.cornell.edu/public/CBN/1996

[10] S. Henderson, Proc. 8th Mtg. of DPF (Albuquerque NM), World Scientific, (1994) 1480.

[11] S. Henderson and S. Roberts, these proceedings.

[12] N. Mistry, Proc. Part. Acc. Conf., 1997, 3559. 